\title{
Congestion Management by Allocating Network Use Cost for the Small-Scale DER Aggregator Market in South Korea
}

\author{
Nadya Noorfatima ${ }^{1}$, Yejin Yang ${ }^{1}$, Jaesung Jung ${ }^{1, *(1)}$ and Jun-Sung Kim ${ }^{2}(\mathbb{D}$ \\ 1 Department of Energy Systems Research, Ajou University, Suwon 16499, Korea; nadyanrf@ajou.ac.kr (N.N.); \\ yangyj@ajou.ac.kr (Y.Y.) \\ 2 Digital Solution Laboratory, Korea Electric Power Research Institute, Daejeon 34056, Korea; \\ kim.junsung@kepco.co.kr \\ * Correspondence: jjung@ajou.ac.kr; Tel.: +82-31-219-2695
}

Citation: Noorfatima, N.; Yang, Y.; Jung, J.; Kim, J.-S. Congestion Management by Allocating Network Use Cost for the Small-Scale DER Aggregator Market in South Korea. Energies 2021, 14, 3524. https:// doi.org/10.3390/en14123524

Academic Editor: Daniela Proto

Received: 20 April 2021

Accepted: 10 June 2021

Published: 13 June 2021

Publisher's Note: MDPI stays neutral with regard to jurisdictional claims in published maps and institutional affiliations.

Copyright: (c) 2021 by the authors. Licensee MDPI, Basel, Switzerland. This article is an open access article distributed under the terms and conditions of the Creative Commons Attribution (CC BY) license (https:// creativecommons.org/licenses/by/ $4.0 /)$.

\begin{abstract}
The increasing penetration level of distributed energy resources (DERs) increases the risk of congestion in the distribution network. To mitigate this, the concept of the small-scale DER aggregator was introduced as a change from uncoordinated to coordinated DERs. However, without appropriate network use cost allocation, the unwanted DER curtailment will be enforced by the network operator. Therefore, this paper proposes a new approach for congestion management by allocating the different network usage costs depending on how much congestion is caused by the DERs in the distribution network. For this, a modified Kirschen's tracing method is proposed and applied to the small-scale DER aggregator market. To verify the effectiveness of the proposed method, a simulation of the small-scale DER aggregator market in South Korea was performed under the IEEE 69-bus distribution network. The model was able to allocate the different network usage costs at different buses and, thus, encouraged the DERs to reduce their generation by charging the energy storage system (ESS) to mitigate congestion. An economic benefit analysis was also performed from the point of view of the aggregator concerning whether they should have an ESS or not.
\end{abstract}

Keywords: distributed energy resource; aggregator; electricity market; congestion management; network cost allocation

\section{Introduction}

The number of distributed energy resources (DERs) has increased in the last decade. However, this increasing integration of DERs has the potential to disrupt the stability and reliability of the power system and thus increase the risk of congestion in the distribution network. A high penetration level of DERs was found to cause an exceeding power flow which yielded congestions and an increase in losses [1]. To mitigate these problems, congestion management was required to manage the electricity supply and demand [2].

The established congestion management is applied to the transmission line by using the optimal power flow (OPF) to regulate the generators based on their generation profile and network constraints [3]. However, the recent DER growth has led to congestion, which is expected to be worsen in the future. To overcome this problem, the technical constraints were considered by curtailing DER generation. However, since a small-scale DER is connected through an inverter, it disconnects the DER from the grid instead of curtailing some of its generation [4]. Furthermore, DERs are owned by different stakeholders in the distribution systems. Thus, establishing a common standard for different stakeholders is complicated. Therefore, small-scale DERs are considered to be regulated by using the electricity price as a cost-effective method [5].

The concept of the small-scale DER aggregator was introduced to shift from uncoordinated to coordinated DERs through the mechanism of the electricity market. In this market model, the aggregator controls the generation patterns by using the price signals determined by the market operator. Thus, the benefit of the DER is determined based 
on the wholesale electricity price and compensation for network usage. However, in the existing small-scale DER aggregator market, the network use for market operation is either charged by a fixed network cost or not compensated at all [6]. Hence, owing to the uniform network cost charged to all DERs, the unwanted and unnecessary DER curtailment is enforced by the network operator even when connected to a bus that does not have any congestion problems [7]. Therefore, to solve this problem, different network use costs (NUCs) should be allocated to the different buses depending on how much the DER causes congestion in the distribution network. This can be carried out using the network cost allocation (NCA) method.

The NCA method can be categorized into two types based on whether it utilizes power flow calculation: non-power flow-based and power flow-based methods. The contribution of each generator unit in the non-power flow-based method is calculated by using the fixed network cost of each line and the magnitude of the DER's transacted power as in postage stamp and contract path methods [8]. Among non-power flow-based methods, the postage stamp method is widely used in some countries because of its simplicity [9-11]. Power flow-based methods, such as proportional sharing, Kirschen's tracing, equivalent bilateral exchange, and Z-Bus NCA methods, identify the amount of power flow on specific lines due to market transactions and thus calculate the contribution of every generation unit to each transaction as the NUC [12]. However, the power flow-based method is more appropriate for the small-scale DER aggregator market because it can identify the contribution of each DER in the power flow of every distribution line to detect the contribution of DERs to network congestion.

Bialek's method proportionally allocates generators' contributions by adopting the power tracing method [13-15]. Nonetheless, among the power flow-based methods, Kirschen's tracing method has advantages over other NCA methods. It can identify the contributions of the DER generation on every line and, at the same time, prevent the allocation of an unexpectedly high NUC to the DER. The basic principle of Kirschen's tracing method uses a traceable equivalent network that can be used for all types of network configuration [16]. However, the application of Kirschen's tracing method in allocating NUCs, which incorporate congestion management through the small-scale DER aggregator market, has not yet been studied.

Therefore, this paper proposes a modification of Kirschen's tracing method to dispatch the schedule of the DERs for congestion management. The composed NUC will encourage the DERs to reduce their generated capacity spontaneously by charging the expensive NUC if they cause congestion. The proposed method was evaluated under the IEEE 69-bus distribution network with the small-scale DER aggregator market in South Korea. The results show that the NUC allocated to the DERs is differentiated according to the DER contribution to the network usage, and thus it can be used to mitigate the congestion. In this case, the energy storage system (ESS) operation schedule is constructed as a response of the DERs towards the high NUC that is imposed on them. Thus, to suggest ESS installation as one of the options for aggregators, economic benefit analysis is investigated to find a worthwhile ESS for solving congestion issues.

The remainder of this study is organized as follows. The small-scale DER aggregator market in South Korea is introduced in Section 2. Then, the modified Kirschen's tracing method is proposed in Section 3. In Section 4, a case study of an aggregator market with various DERs, including small-scale PVs and ESSs, is presented. Then, the simulation results are discussed and analyzed to demonstrate the effectiveness of the proposed method. Finally, conclusions and a scope for future research are presented.

\section{Small-Scale DER Aggregator Market in South Korea}

This section describes the small-scale DER aggregator market in South Korea. There are two ways to integrate small-scale DERs: through the power purchase agreement (PPA) contract, and through the Korea Power Exchange (KPX) aggregator market [17]. The PPA is a contract-based renewable energy generation transaction between DERs and the 
utility company, Korea Power Corporation (KEPCO). According to this contract, all of the generated capacity is purchased by the utility company and compensated by the monthly average system marginal price (SMP), $\overline{S M P}$. The purchased settlement for the DER at time $t$ is formulated as follows:

$$
\text { Total Payment }{ }_{i, t}=\left(P_{i, t} \cdot \overline{S M P}\right),
$$

where $P_{i, t}$ is the metered generation of the DER $i$ at time $t$. Although this contract is simple and easy to integrate small-scale DERs, it has drawbacks regarding congestion issues because DER generation is not regulated and limited owing to the uniform settlement price. Therefore, to reduce the congestion risk, small-scale DER generation management through the aggregator market was introduced in South Korea [18].

In the KPX small-scale DER aggregator market, an additional incentive is provided if the DER helps to mitigate unexpected congestion due to the uncertainties of renewable energy generations. The incentive is calculated based on three components: the metered generation of the DER $i$ at time $t\left(P_{i, t}\right)$, the incentive unit price $(\rho)$, and the constraint satisfaction flag $(\alpha)$. The incentive $I_{i, t}$ and total payment at time $t$ are given to the DER $i$ according to the following equations:

$$
\begin{gathered}
I_{i, t}=P_{i, t} \cdot \rho \cdot \alpha, \\
\text { Total Payment }_{i, t}=P_{i, t} \cdot S M P_{t}+I_{t},
\end{gathered}
$$

where $\rho$ is the applied $3 \mathrm{KRW} / \mathrm{kWh}$ and $4 \mathrm{KRW} / \mathrm{kWh}$ if the error rate is within 6 to $8 \%$ and less than $6 \%$, respectively. $\alpha$ is an indicator that determines whether the participant can receive the incentive. If the error rate is greater than $8 \%, \alpha$ is 0 . Otherwise, $\alpha$ is set to 1. Furthermore, this incentive is only provided when the DER output is greater than $10 \%$ of the installed capacity. The error rate quantifies the difference between the forecasted generation (bidding) and the metered generation.

However, the error rate could be inevitably high owing to the intermittent renewable generations. According to [19], the application of an ESS could reduce the error rate because it is able to control the power output by charging and discharging energy to meet the forecasted generation. However, further compensation for ESS application in the small-scale DER aggregator market has not yet been discussed. It is worthwhile if an ESS is able to make enough profit in the market compared with the investment of the ESS.

Furthermore, the South Korean DER aggregator market exempts payment for network usage. However, the KPX is planning to charge an NUC and apply the fixed NUC $\left(\lambda_{F}\right)$ in Table 1. Therefore, we assume that the total profit will be calculated after deducting the fixed NUC from the total payment as follows:

$$
\text { Total Profit } t_{i, t}=\text { Total Payment } t_{i, t}-P_{i, t} \cdot \lambda_{F}
$$

Table 1. Fixed network use cost $\left(\lambda_{F}\right)$.

\begin{tabular}{ccc}
\hline Category & Basic Charge & Energy Charge \\
\hline Below $600 \mathrm{~V}$ & $914 \mathrm{KRW} / \mathrm{kW}$ & $9.43 \mathrm{KRW} / \mathrm{kWh}$ \\
Over 600 V & $890 \mathrm{KRW} / \mathrm{kW}$ & $3.13 \mathrm{KRW} / \mathrm{kWh}$ \\
\hline
\end{tabular}

\section{The Proposed NCA Method}

In this section, the NCA method is proposed to mitigate the congestion induced by small-scale DERs. The proper allocation of the NUC prevents unexpected congestion.

\subsection{Kirschen's Power Tracing Method}

In this method, the buses and branches of the network are represented as the domain of a generator and common depending on which generator supplied the power [12]. Initially, the domain of a generator is determined as the set of buses that are supplied by the same 
generator according to the direction of the power flow. However, the concept of the domain of a generator alone is insufficient to determine the amount of power supplied by each generator if multiple generators supply power to the same bus. Therefore, the common method is used to classify the contagious buses supplied by the same generator. Separated sets of buses supplied by the same generators are identified as independent commons.

Then, the commons are ranked to construct an equivalent network. A common is ranked highest ( $r a n k=1$ ) if the common is supplied by the lowest number of generators. On the other hand, if a common is supplied by many generators, it has a lower rank. The rank determines the position of the commons in the equivalent network. The power flows from the common with the highest rank to the lower-rank commons. Using the direction of the power flows, links between commons are formed. Finally, the equivalent network is constructed as an arrangement of commons and links.

To calculate the contribution of each generator to the commons and line flows, the equivalent network is analyzed using two types of contributions: absolute and relative contributions. The absolute contribution is the total inflow at the link to common $s$ due to generator $i\left(A_{i, s}\right)$. The relative contribution $\left(R_{i, s}\right)$ illustrates the proportion of generator $i$ with respect to total flows at the link to common $s$.

The contributions are determined sequentially from the highest rank to lower-rank commons. Initially, the inflows of all commons are calculated as the accumulation of flow at links from common $r$ toward common $s\left(F_{r, s}\right)$, which is obtained from the power flow results. Then, for the highest ranked common, the absolute contribution is equal to the capacity of the generator that is directly connected. Thus, according to Equation (6), the relative contribution of the first rank common will be equal to 1 . For the lower-rank commons, $A_{i, s}$ and $R_{i, s}$ are calculated as follows:

$$
\begin{gathered}
A_{i, s}=\sum_{r} R_{i, r} \cdot F_{r, s}, \\
R_{i, s}=\frac{A_{i, s}}{\sum_{r} F_{r, s}},
\end{gathered}
$$

where $R_{i, r}$ is the relative contribution of generator $i$ to the higher-rank common $r$. Furthermore, if the accumulation of the relative contribution with respect to each bus is not equal to 1 , then the error of power tracing occurs, which can be identified from the mismatch common [20]. The absolute and relative contributions of each generator $i$ at each branch in the real network can be calculated. By identifying the connected common to the branch, the total contribution of generator $i$ to the power flow on branch $k\left(F_{i, k}\right)$ is calculated by the given formula.

$$
F_{i, k}=R_{i, r} \cdot F_{k}
$$

where $F_{k}$ is the power flows at branch $k$. The overall flowchart of Kirschen's power tracing method is shown in Figure 1.

\subsection{Sensitivity Index}

According to [21], congestion occurs when a large generation of a DER cannot be consumed by the local area but a distant location. Under these conditions, the remaining reverse power flow, which is not consumed by the local area, causes an increase in the network losses and a decrease in operational efficiency, including overloading and voltage violations in the distribution network [22]. Therefore, to mitigate congestion, it is important to identify the contribution of DER generation to the change in the power flow at various locations under reverse flow. 


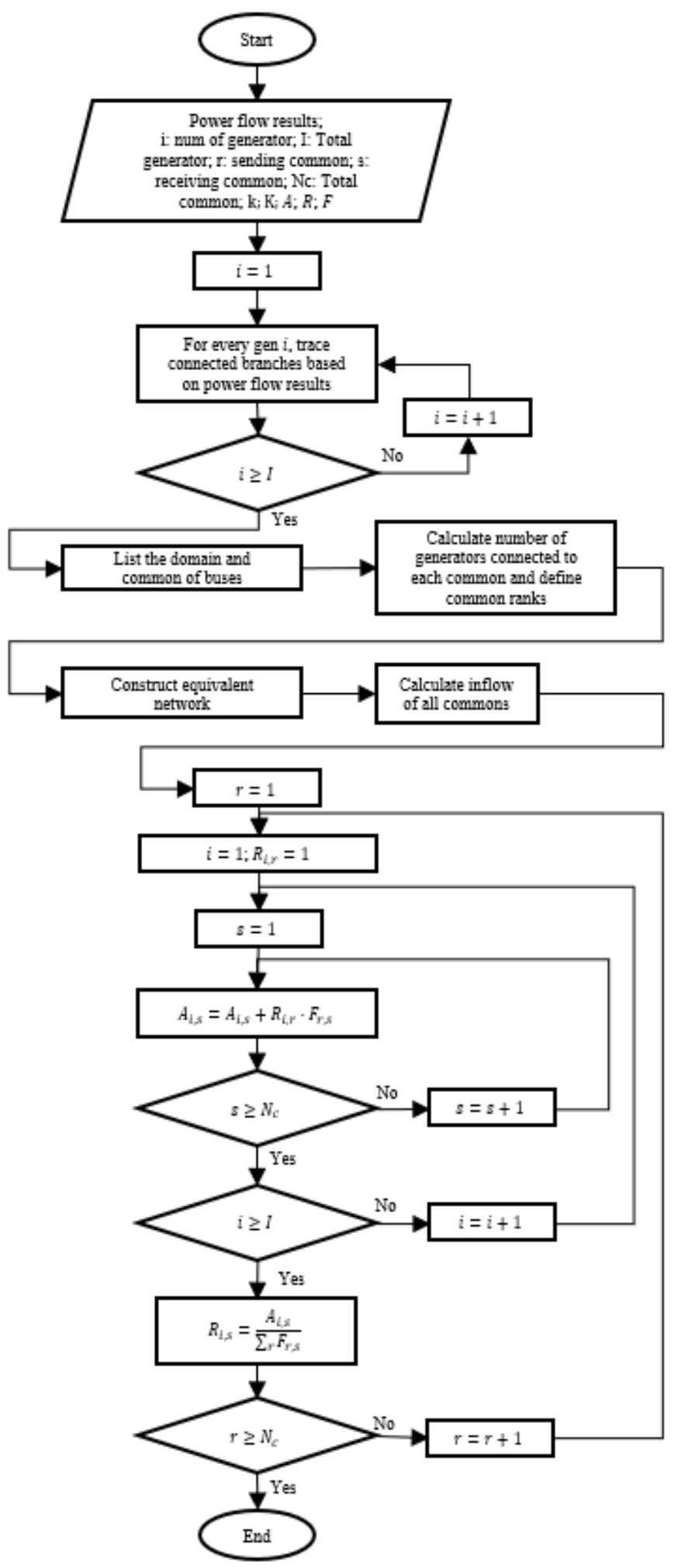

Figure 1. Flowchart of Kirschen's power tracing method.

To evaluate the effectivity of a generator's change in impacting the power flow, the sensitivity index was employed in this paper [23]. Although it is used for security analysis of a power system, it can also be applied to evaluate the congestion risk of DERs in the distribution network. To determine the sensitivity index, the relation between the change 
in generation $\left(\Delta P_{i}\right)$ and the change in power flow on each branch $k\left(\Delta F_{k}\right)$ was required. The relation is expressed by using a sensitivity factor that is calculated as follows:

$$
\xi_{k}^{i}=\frac{\Delta F_{k}}{\Delta P_{i}}
$$

where $\xi_{k}^{i}$ represents the sensitivity factor of generator $i$ in branch $k$. The change in generation $\left(\Delta P_{i}\right)$ can be the fixed amount or some proportion of the DER generation to calculate the factor.

Then, the sensitivity index was calculated to identify the normalized effectivity of the change in DER generation, which can be different in size and located in various locations. To determine the sensitivity index, the sensitivity factor was scaled using the following formula:

$$
\delta_{k}^{i}= \begin{cases}1 & ; \xi_{k}^{i} \geq \xi^{\max } \\ \frac{\xi_{k}^{i}-\xi^{\min }}{\xi^{\max }-\xi^{\min }} & ; \xi^{\min }<\xi_{k}^{i}<\xi^{\max } \\ 0 & ; \xi_{k}^{i} \leq \xi^{\min }\end{cases}
$$

where $\delta_{k}^{i}$ is the sensitivity index of generation $i$ in branch $k$. $\xi^{\text {max }}$ and $\xi^{\text {min }}$ represent the maximum and minimum sensitivity factors in the system, respectively, which are determined as the highest and lowest sensitivity factors of all DERs among every branch of the system, respectively. The sensitivity index determines whether the generation change will effectively affect the change in the power flow. The DER with the most impact has the highest sensitivity index, " 1 ". In contrast, a DER with a lower sensitivity has less impact on the power flow.

\subsection{Modified NUC Allocation of Kirschen's Power Tracing Method}

In the original Kirschen's tracing method, after the contribution of all generators is determined, the NUC is calculated by the following formula:

$$
\lambda_{i, k}=\frac{F_{i, k}}{\sum_{k}^{K} \sum_{i}^{I} F_{i, k}} \cdot C,
$$

where $C$ is the total network operating cost. According to Equation (10), the basic principle of the NUC allocation of Kirschen's tracing method is based on the proportion of the flow supplied by each generator in each branch power flow. Hence, a DER with a high generation capacity will charge a high NUC even though its generation is consumed by the local area and, thus, does not contribute to congestion. Therefore, in this section, Kirschen's tracing method for NUC allocation is modified by using a sensitivity index to distribute the NUC according to the generators' contribution to congestion.

Furthermore, the modified NUC allocation of Kirschen's tracing method is designed to compensate for the cost reduction of network operation owing to small-scale DER integration. Therefore, the network operator maintains the network operating cost so that it cannot hinder the integration of the DER because of the shortage of income. Thus, the modified method distributes the operating cost reduction to the small-scale DER depending on the potential congestion risk.

The modified NUC allocation using Kirschen's tracing method is shown in Figure 2. As described in the previous section, the sensitivity factor of each generator was scaled to obtain the sensitivity index. To calculate the sensitivity factor, $\Delta F_{k}$ and $\Delta P_{i}$ were determined. If $\Delta P_{i}$ is adjusted initially, $\Delta F_{k}$ can be determined by using Kirschen's tracing method. 


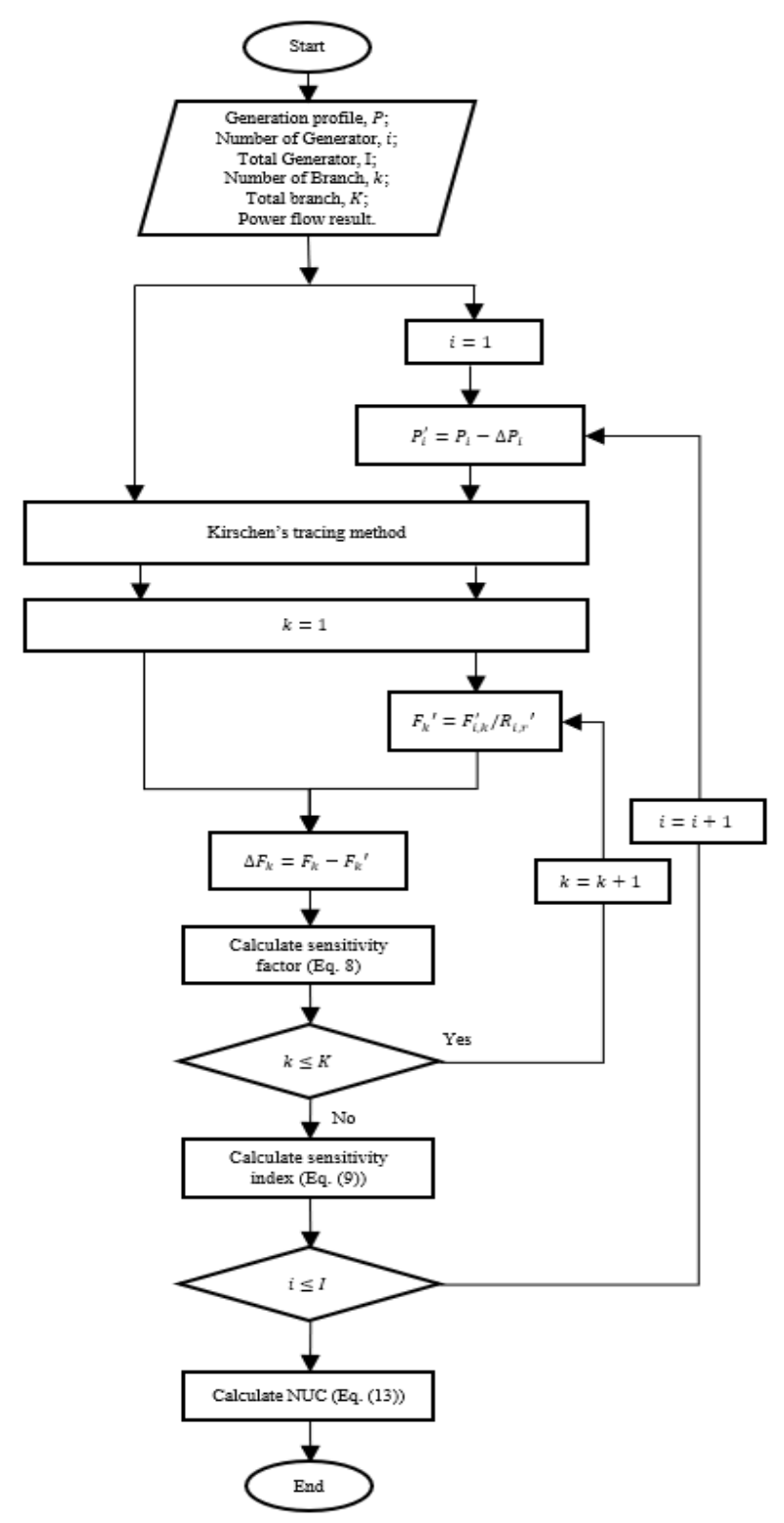

Figure 2. Proposed NCA method using a modified Kirschen's power tracing method with sensitivity index.

To calculate $\Delta F_{k}$, Kirschen's tracing method is applied twice: once using the original generation capacity and then with the changed generation capacity. First, Kirschen's tracing method is performed based on the power flow results of the original generation capacity to determine the $F_{i, k}$ for the NUC calculation. Then, Kirschen's tracing method is repeated to determine the changed power flow at branch $k\left(F_{k}{ }^{\prime}\right)$ due to the changed generation capacity of generator $i\left(P_{i}^{\prime}\right)$. The $F_{k}{ }^{\prime}$ and $P_{i}^{\prime}$ are formulated as follows:

$$
\begin{gathered}
F_{k}{ }^{\prime}=\frac{F_{i, k}^{\prime}}{R_{i, r^{\prime}}}, \\
P_{i}^{\prime}=P_{i}-\Delta P_{i},
\end{gathered}
$$

where $F_{k}{ }^{\prime}$ is the changed power flow on branch $k ; F_{i, k}^{\prime}$ represents the contribution of generator $i$ to the power flow at branch $k ; R_{i, r}{ }^{\prime}$ is the updated relative contribution of generator $i$ to common $r$. Due to the $P_{i}{ }^{\prime}$, the generation capacity at the swing bus should be adjusted to maintain the system balance. The power flows at several locations are changed. 
In this case, the contribution to power flows on some branches and the relative contribution of each generator can be updated eventually.

After determining the sensitivity factor of all the branches, the sensitivity index of the generator is determined using Equation (9). This is conducted by changing the generation capacity of generator $i$, once every time. After this is conducted for all the generators, the NUC of each generator $i$ for every branch $k\left(\lambda_{i, k}\right)$ is calculated by using Equation (13). The sensitivity index was incorporated in the calculation as the weighting factor. Thus, the total NUC allocated to each generator $i\left(\lambda_{i}\right)$ to compensate the network usage is formulated as Equation (14).

$$
\begin{gathered}
\lambda_{i, k}=\left(\frac{F_{i, k} \cdot\left(1+\delta_{k}^{i}\right)}{\sum_{k}^{K} \sum_{i}^{I} F_{i, k} \cdot\left(1+\delta_{k}^{i}\right)}\right) \cdot C \\
\lambda_{i}=\sum_{k}^{K} \lambda_{i, k}
\end{gathered}
$$

By assigning $\delta_{k}^{i}$ to $F_{i, k}$ in the NUC calculation, the generators' contribution to congestion determines the NUC allocated to them. A generator with a higher sensitivity index will have a higher proportion of total compensation, and thus it will be imposed with a high NUC. If the sensitivity index of a generator at a certain branch is low, the NUC allocated to that generator will be relatively low. Thus, a DER will not have to pay for a high NUC if it supplies a large generation unless it contributes to congestion.

\section{Results and Discussion}

The verification of the proposed NUC method using the IEEE 69-bus distribution test system is described in this section [24]. To analyze the effect of congestion management, the proposed method is compared with a fixed NUC method. Furthermore, an economic analysis is performed from the point of view of the aggregator to evaluate whether the installation of an ESS is beneficial.

\subsection{Case Study}

A distribution system containing multiple DERs was simulated based on the smallscale DER aggregator market operation in South Korea. For this, the original IEEE test system was modified to incorporate multiple loads and DERs depending on whether the customer type is industrial, commercial, or residential, as shown in Figure 3.

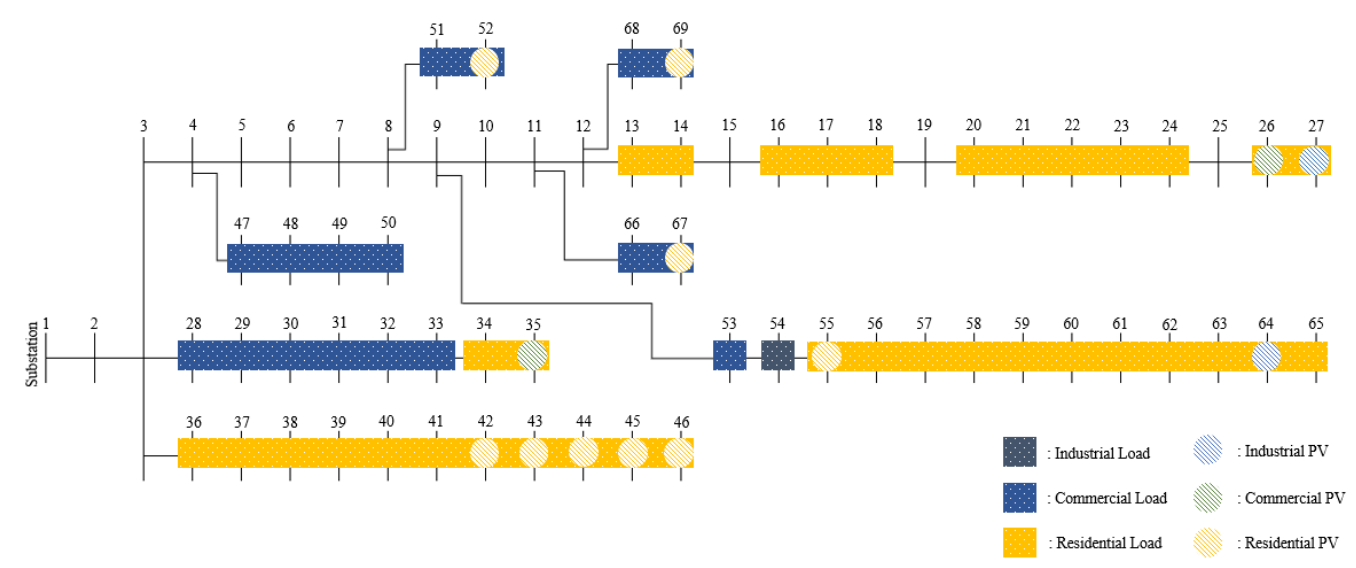

Figure 3. IEEE 69-bus distribution system.

The locations of the applied loads and DERs are listed in Table 2. The capacities of the residential, commercial, and industrial loads are below $500 \mathrm{~kW}$, below $1 \mathrm{MW}$, and more than $1 \mathrm{MW}$, respectively. Furthermore, the capacities of residential, commercial, and industrial PVs are $200 \mathrm{~kW}$, below $2 \mathrm{MW}$, and greater than $2 \mathrm{MW}$. 
Table 2. Load and DER location by the customer types in test system.

\begin{tabular}{|c|c|c|}
\hline \multirow{2}{*}{ Category } & \multicolumn{2}{|c|}{ Locations (Bus) } \\
\hline & Load & DER \\
\hline Residential & $13-14 ; 16-18 ; 20-24 ; 26-27$ & $\begin{array}{c}\text { DER 5-9: 42-46; DER 10: 52; DER 11: 55; } \\
\text { DER 12: 67; DER 13: } 69\end{array}$ \\
\hline Commercial & $28-33 ; 47-50 ; 51-53 ; 66-69$ & DER 1: 26; DER 4: 35 \\
\hline Industrial & 54 & DER 2: 27; DER 3: 64 \\
\hline
\end{tabular}

The original test system did not provide a time-varying load; therefore, simulations were performed using the hourly consumption data obtained from [25]. For diversity, different types of loads are applied, including industrial, commercial, and residential customers. Figure 4 shows their normalized patterns of load profiles during the day. It can be seen that the residential customers have no significant loading variation throughout the day. However, the electricity consumption of commercial and industrial customers depends on their business hours.

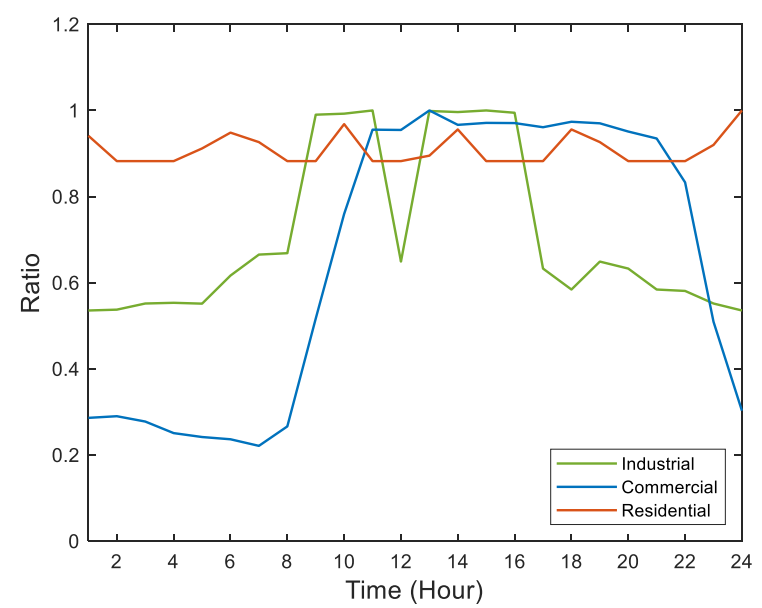

Figure 4. Normalized load profiles by customer types on a selected day.

For a more realistic simulation, the actual time-varying PV generation data on 22 May 2020 were obtained from PVWatts [26]. Figure 5 shows the corresponding normalized PV generation profiles. It can be seen that the generation peaks at 1 p.m. on a selected day.

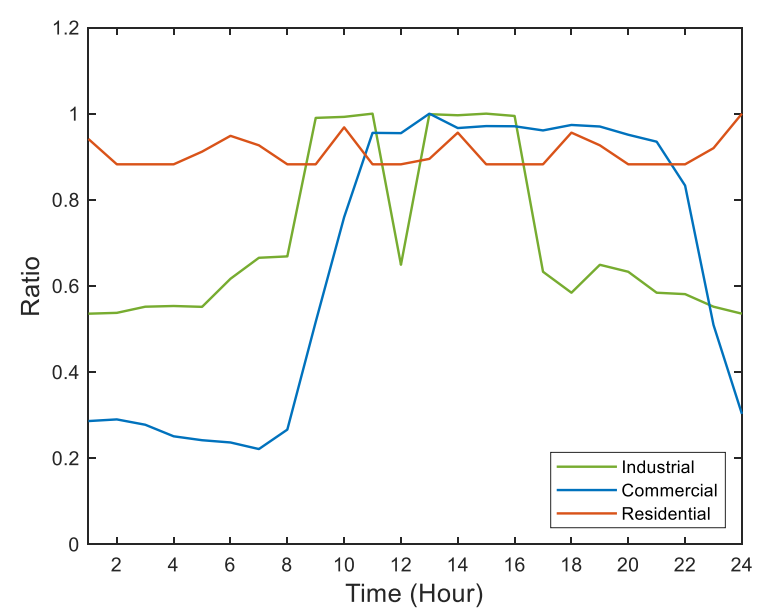

Figure 5. Normalized PV generation profile. 
In addition, to calculate the aggregator's small-scale DER market profit, the actual hourly SMP data on 22 May 2020 were used, as shown in Figure 6 [27]. During the weekday, a greater SMP is observed from 9 a.m. to 11 a.m. and after 4 p.m. than at other times.

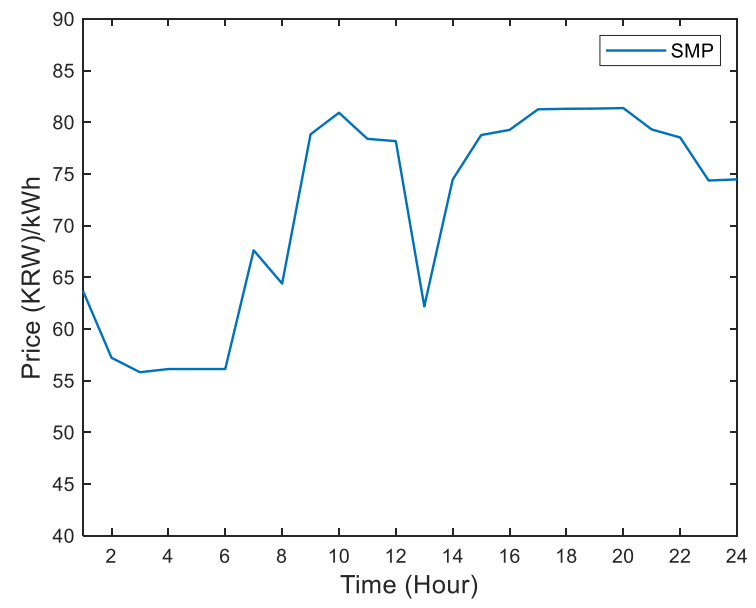

Figure 6. System marginal price.

\subsection{Kirschen's Tracing Method Equivalent Network}

To construct the Kirschen's tracing method equivalent network, a power flow calculation was required to obtain the flows on each branch of the system. The detail of the power flow algorithm was not considered in this paper; thus, MATPOWER was used for the calculation [28]. Based on the results, the commons of the buses are listed in Table 3. Then, the ranks of commons are defined to construct the equivalent network. As there are 14 generators (one substation and 13 DERs) in the test system, the maximum possible number of ranks is 14 . According to the number of generators that supply each common, six ranks are observed in the test system.

Table 3. List of commons and ranks in the test system.

\begin{tabular}{cccccc}
\hline Common & Bus & Rank & Common & Bus & Rank \\
\hline 1 & $1-8$ & 1 & 12 & 52 & 2 \\
2 & 27 & 1 & 13 & 55 & 2 \\
3 & $28-31$ & 1 & 14 & 9 & 3 \\
4 & $33-35$ & 1 & 15 & 43 & 3 \\
5 & $36-41 ; 47-51$ & 1 & 16 & 53 & 3 \\
6 & $56-65$ & 1 & 17 & 66 & 3 \\
7 & 67 & 1 & 18 & 68 & 3 \\
8 & 69 & 1 & 19 & 44 & 4 \\
9 & $10-26$ & 2 & 20 & 45 & 5 \\
10 & 32 & 2 & 21 & 54 & 5 \\
11 & 42 & 2 & 22 & 46 & 6 \\
\hline
\end{tabular}

From these data, an equivalent network was constructed. For simplicity, the equivalent network of the generators is presented separately, and only some representative generators are shown in Figure 7. To construct the equivalent network, the power flows from the substation were initially traced according to the power flow results. Then, the commons of the buses connected to each generator were identified. For example, the power flow results show that the substation supplies buses 1 to 8 . Then, according to the common list, buses 1 to 8 are identified as common 1 . Furthermore, the first bus connected to DER 1 is bus 26; thus, according to the common lists, bus 26 is identified as common 9. Then, with respect to the power flow results, the remaining connected buses from the substation and DER 1 are traced, and thus their commons are identified. 


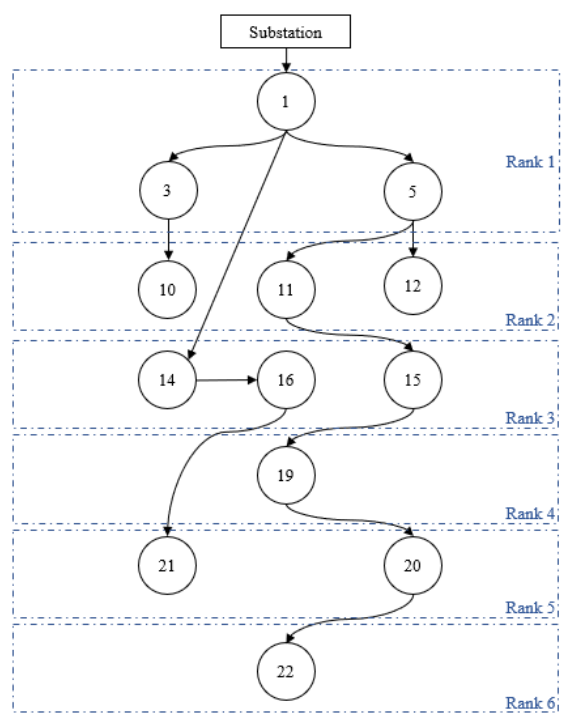

(a)

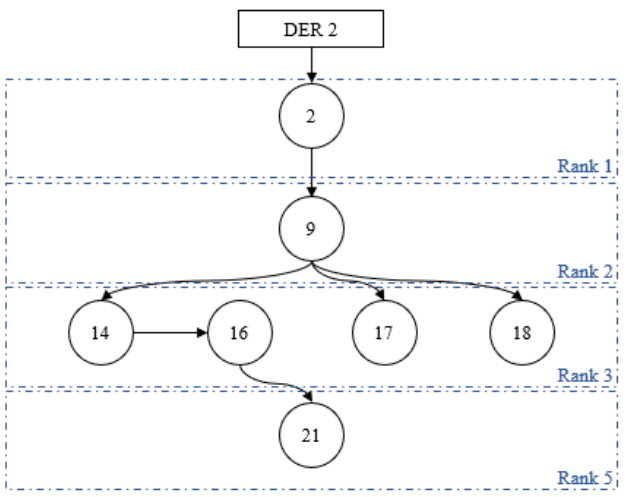

(c)

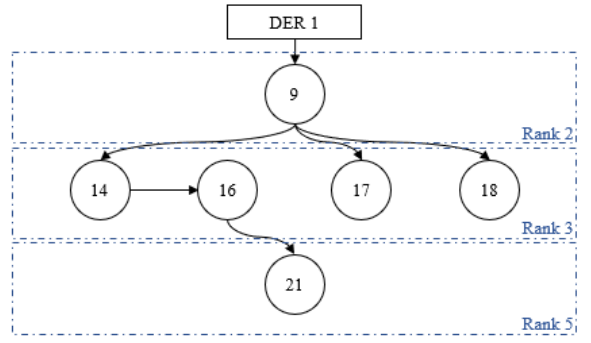

(b)

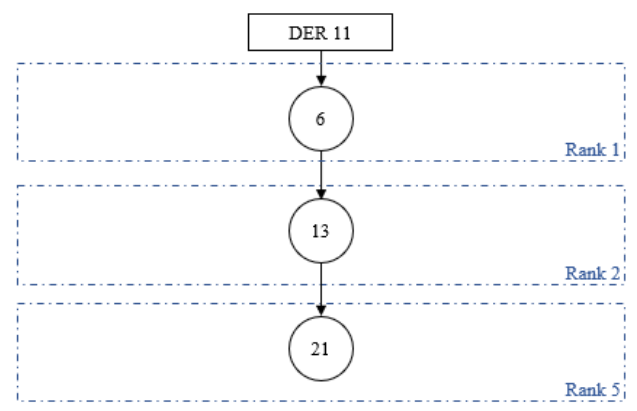

(d)

Figure 7. Equivalent network of the (a) substation; (b) DER 1; (c) DER 2; (d) DER 11.

After all connected commons are identified, they are configured based on their ranks and connected to each other according to the power flow directions represented as links. Finally, the equivalent networks of the substation and DER 1 are illustrated in Figure 7a,b. The equivalent networks of DER 2 and DER 11 are also presented in Figure 7c,d, respectively.

Kirschen's tracing method is mainly used to determine the contributions of each generator to the power flows so that the amount of network usage of each generator can be determined. Table 4 shows the relative contributions of the commons. The total contribution of the generators supplying each common should be equal to one. 
Table 4. The relative contribution of generators to commons.

\begin{tabular}{|c|c|c|c|c|c|c|c|c|c|c|c|c|c|c|}
\hline \multirow{2}{*}{ Common } & \multirow{2}{*}{ Substation } & \multicolumn{13}{|c|}{ Generator (DER Number) } \\
\hline & & 1 & 2 & 3 & 4 & 5 & 6 & 7 & 8 & 9 & 10 & 11 & 12 & 13 \\
\hline 1 & 1 & 0 & 0 & 0 & 0 & 0 & 0 & 0 & 0 & 0 & 0 & 0 & 0 & 0 \\
\hline 2 & 0 & 0 & 1 & 0 & 0 & 0 & 0 & 0 & 0 & 0 & 0 & 0 & 0 & 0 \\
\hline 3 & 1 & 0 & 0 & 0 & 0 & 0 & 0 & 0 & 0 & 0 & 0 & 0 & 0 & 0 \\
\hline 4 & 0 & 0 & 0 & 0 & 1 & 0 & 0 & 0 & 0 & 0 & 0 & 0 & 0 & 0 \\
\hline 5 & 1 & 0 & 0 & 0 & 0 & 0 & 0 & 0 & 0 & 0 & 0 & 0 & 0 & 0 \\
\hline 6 & 0 & 0 & 0 & 0 & 0 & 0 & 0 & 0 & 0 & 0 & 0 & 1 & 0 & 0 \\
\hline 7 & 0 & 0 & 0 & 0 & 0 & 0 & 0 & 0 & 0 & 0 & 0 & 0 & 1 & 0 \\
\hline 8 & 0 & 0 & 0 & 0 & 0 & 0 & 0 & 0 & 0 & 0 & 0 & 0 & 0 & 1 \\
\hline 9 & 0 & 0.3814 & 0.6186 & 0 & 0 & 0 & 0 & 0 & 0 & 0 & 0 & 0 & 0 & 0 \\
\hline 10 & 0.7132 & 0 & 0 & 0.2868 & 0 & 0 & 0 & 0 & 0 & 0 & 0 & 0 & 0 & 0 \\
\hline 11 & 0.1869 & 0 & 0 & 0 & 0.8131 & 0 & 0 & 0 & 0 & 0 & 0 & 0 & 0 & 0 \\
\hline 12 & 0.1736 & 0 & 0 & 0 & 0 & 0 & 0 & 0 & 0 & 0.8264 & 0 & 0 & 0 & 0 \\
\hline 13 & 0 & 0 & 0 & 0 & 0 & 0 & 0 & 0 & 0 & 0 & 0.3282 & 0.6718 & 0 & 0 \\
\hline 14 & 0.2867 & 0.2721 & 0.4413 & 0 & 0 & 0 & 0 & 0 & 0 & 0 & 0 & 0 & 0 & 0 \\
\hline 15 & 0.1671 & 0 & 0 & 0 & 0.0996 & 0.7333 & 0 & 0 & 0 & 0 & 0 & 0 & 0 & 0 \\
\hline 16 & 0.2867 & 0.2721 & 0.4413 & 0 & 0 & 0 & 0 & 0 & 0 & 0 & 0 & 0 & 0 & 0 \\
\hline 19 & 0.1503 & 0 & 0 & 0 & 0.0896 & 0.0952 & 0.6649 & 0 & 0 & 0 & 0 & 0 & 0 & 0 \\
\hline 20 & 0.1216 & 0 & 0 & 0 & 0.0724 & 0.0770 & 0.0813 & 0.6477 & 0 & 0 & 0 & 0 & 0 & 0 \\
\hline 21 & 0.2511 & 0.2383 & 0.3865 & 0 & 0 & 0 & 0 & 0 & 0 & 0 & 0.0407 & 0.0834 & 0 & 0 \\
\hline 22 & 0.1003 & 0 & 0 & 0 & 0.0598 & 0.0635 & 0.0671 & 0.1579 & 0.5514 & 0 & 0 & 0 & 0 & 0 \\
\hline
\end{tabular}




\subsection{NUC Allocation Results}

In this section, the NUC allocation to the DERs using the proposed method is compared with that using the fixed NUC and postage stamp methods and Bialek's method. The simulation was performed at the PV peak time (1 p.m.). Large PV generation can cause congestion problems represented by voltage violations unless a proper dispatch is applied. The voltage violation is represented when the voltage magnitude exceeds $\pm 10 \%$ of the nominal value [29]. Figure 8 shows the location of a voltage violation occurring because of a large PV generation injected into the system at peak time as a reference case. However, this congestion problem cannot be released by the fixed NUC charge because it only considers the amount of generation. Therefore, the NUC needs to be charged differently depending on the significance of causing a congestion problem.

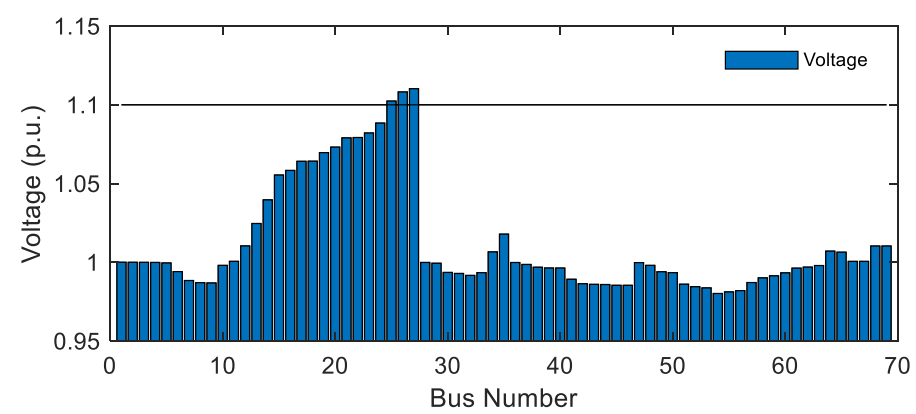

Figure 8. Voltage profile at the buses.

Furthermore, the proposed NUC method is designed to allocate the total network operational cost based on the contribution of the substation and DER to congestion. Therefore, the network operator maintains its operating cost to support stable network operation even under the condition of high penetration of small-scale DERs. If the total network operational cost is known, it can be directly used. However, it is unknown here. Thus, in this paper, the total network operational cost is assumed as the multiplication of the total transacted energy capacity and the fixed NUC per $\mathrm{kWh}$ to maintain the system. In the test system, the total power flow at the distribution system without DERs is 20,719.64 kWh, which requires a total of KRW $64,852.48(20,719.64 \mathrm{kWh} \times 3.13 \mathrm{KRW} / \mathrm{kWh})$. Therefore, the amount $C$ (KRW 64,852.48) in Equation (13) must be distributed to the substation and all DERs.

Table 5 shows a comparison of the NUC allocation results. In the fixed NUC case, the NUC is allocated to all generators uniformly depending on their capacity. However, the NUC obtained from the proposed method is different depending on the significance of the congestion problem caused by the substation and DERs. This reduces the total network operational cost but maintains the average NUC at its reference case value. Nevertheless, in the proposed NUC method, the total network operational cost is maintained as expected but the average NUC is greater than the fixed NUC. This is because the introduction of DERs reduces the delivery loss and, thus, reduces the total required generation in the system.

In Figure 8, bus 25 to bus 27 show overvoltage violations due to the reverse flow injected from DER 1 and DER 2. Thus, DER 1 and DER 2 are charged with a greater NUC compared to other DERs. Furthermore, DER 11, which has the same generation capacity as DER 2, is charged with a lower NUC than DER 2 because of the large load connected at the same bus with DER 11. It is consumed locally and thus does not cause significant congestion issues. However, in the fixed NUC case, this method charges the same NUC regardless of the congestion issues.

Furthermore, the proposed method is compared with other NCA methods, including the postage stamp method and Bialek's method, to verify its results and highlight its advantages. Using the postage stamp method, the NUC allocated to each generator is fixed like the fixed NUC, but the value is the same as the average of the proposed NUC method 
because the total network operational cost is the same as the proposed NUC method. On the other hand, Bialek's method obtains a different NUC allocated to each DER. However, the NUC allocated to DER 2 is much smaller than that allocated to DER 1, even though both induce congestion problems. In addition, the NUC allocated to DER 11 is higher than that allocated to DER 2, even though DER 11 does not induce any congestion problems.

Table 5. NUC allocation results comparison.

\begin{tabular}{|c|c|c|c|c|c|c|c|c|}
\hline \multirow{2}{*}{$\begin{array}{c}\text { Generator } \\
\text { (DER } \\
\text { Number) }\end{array}$} & \multicolumn{2}{|c|}{ Fixed NUC } & \multicolumn{2}{|c|}{ Postage Stamp } & \multicolumn{2}{|c|}{ Bialek's Method } & \multicolumn{2}{|c|}{ Proposed } \\
\hline & $\begin{array}{c}\text { Total NUC } \\
\text { (KRW) }\end{array}$ & $\begin{array}{c}\text { NUC } \\
\text { (KRW/kWh) }\end{array}$ & $\begin{array}{c}\text { Total NUC } \\
\text { (KRW) }\end{array}$ & $\begin{array}{c}\text { NUC } \\
\text { (KRW/kWh) }\end{array}$ & $\begin{array}{c}\text { Total NUC } \\
\text { (KRW) }\end{array}$ & $\begin{array}{c}\text { NUC } \\
\text { (KRW/kWh) }\end{array}$ & $\begin{array}{c}\text { Total NUC } \\
\text { (KRW) }\end{array}$ & $\begin{array}{c}\text { NUC } \\
\text { (KRW/kWh) }\end{array}$ \\
\hline Substation & $38,921.11$ & 3.13 & $39,462.95$ & 3.17 & $37,288.34$ & 2.99 & $35,477.15$ & 2.85 \\
\hline 1 & 3892.37 & 3.13 & 3946.56 & 3.17 & $20,291.4$ & 16.31 & 8109.37 & 6.52 \\
\hline 2 & 6357.54 & 3.13 & 6446.05 & 3.17 & 923.37 & 0.45 & $16,440.57$ & 8.09 \\
\hline 3 & 3892.37 & 3.13 & 3946.56 & 3.17 & 1190.47 & 0.98 & 1088.24 & 0.88 \\
\hline 4 & 504.56 & 3.13 & 511.59 & 3.17 & 563.17 & 3.49 & 185.40 & 1.15 \\
\hline 5 & 504.56 & 3.13 & 511.59 & 3.17 & 633.47 & 3.93 & 123.34 & 0.77 \\
\hline 6 & 504.56 & 3.13 & 511.59 & 3.17 & 299.37 & 1.86 & 56.13 & 0.35 \\
\hline 7 & 504.56 & 3.13 & 511.59 & 3.17 & 334.41 & 2.07 & 62.96 & 0.39 \\
\hline 8 & 504.56 & 3.13 & 511.59 & 3.17 & 0 & 0 & 0 & 0 \\
\hline 9 & 504.56 & 3.13 & 511.59 & 3.17 & 0 & 0 & 0 & 0 \\
\hline 10 & 504.56 & 3.13 & 511.59 & 3.17 & 177.74 & 1.10 & 55.89 & 0.35 \\
\hline 11 & 6357.54 & 3.13 & 6446.05 & 3.17 & 3082.26 & 1.52 & 3190.65 & 1.57 \\
\hline 12 & 504.56 & 3.13 & 511.59 & 3.17 & 34.23 & 0.21 & 31.38 & 0.19 \\
\hline 13 & 504.56 & 3.13 & 511.59 & 3.17 & 34.23 & 0.21 & 31.38 & 0.19 \\
\hline \multirow{2}{*}{ Results } & Total & Mean & Total & Mean & Total & Mean & Total & Mean \\
\hline & $63,962.04$ & 3.13 & $64,852.48$ & 3.17 & $64,852.48$ & 3.17 & $64,852.48$ & 3.17 \\
\hline
\end{tabular}

Furthermore, Table 6 shows a comparison of the allocation factor of each NCA method. The allocation factor represents the level of accentuation of each generator contributing to the network cost [30]. It shows that the proposed method allocates the lowest total NUC to the substation compared with other NCA methods. In contrast, the proposed method allocates a higher NUC to DER 1 and DER 2 compared to DER 3 and DER 11, even though they have similar generation amounts. According to these results, the proposed method is able to identify DERs that cause congestion in the distribution network and thus charge them a greater NUC. Therefore, the proposed method can be used to manage DERs for congestion management, which cannot be performed using other NCA methods. The DERs with a high NUC would voluntarily reduce their generation by curtailment or shift it by an ESS so that it helps to mitigate the congestion problems by the high penetration of DERs in the distribution system.

Table 6. Allocation factors of NCA methods.

\begin{tabular}{|c|c|c|c|c|c|c|c|c|}
\hline \multirow{3}{*}{$\begin{array}{c}\text { Generator } \\
\text { (DER } \\
\text { Number) }\end{array}$} & \multicolumn{8}{|c|}{ Allocation Factors (\%) } \\
\hline & \multicolumn{2}{|c|}{ Fixed NUC } & \multicolumn{2}{|c|}{ Postage Stamp } & \multicolumn{2}{|c|}{ Bialek's Method } & \multicolumn{2}{|c|}{ Proposed } \\
\hline & Total NUC & NUC & Total NUC & NUC & Total NUC & NUC & Total NUC & NUC \\
\hline Substation & 60.85 & 100 & 60.85 & 100 & 57.50 & 94.60 & 54.70 & 89.91 \\
\hline 1 & 6.09 & 100 & 6.09 & 100 & 31.29 & 514.73 & 12.50 & 205.68 \\
\hline 2 & 9.94 & 100 & 9.94 & 100 & 1.42 & 14.34 & 25.35 & 255.21 \\
\hline 3 & 6.09 & 100 & 6.09 & 100 & 1.84 & 30.20 & 1.68 & 27.76 \\
\hline 4 & 0.79 & 100 & 0.79 & 100 & 0.87 & 110.21 & 0.29 & 36.28 \\
\hline 5 & 0.79 & 100 & 0.79 & 100 & 0.98 & 123.96 & 0.19 & 24.29 \\
\hline 6 & 0.79 & 100 & 0.79 & 100 & 0.46 & 58.58 & 0.09 & 11.04 \\
\hline 7 & 0.79 & 100 & 0.79 & 100 & 0.52 & 65.44 & 0.10 & 12.30 \\
\hline 8 & 0.79 & 100 & 0.79 & 100 & 0.00 & 0.00 & 0.00 & 0.00 \\
\hline 9 & 0.79 & 100 & 0.79 & 100 & 0.00 & 0.00 & 0.00 & 0.00 \\
\hline 10 & 0.79 & 100 & 0.79 & 100 & 0.27 & 34.78 & 0.09 & 11.04 \\
\hline 11 & 9.94 & 100 & 9.94 & 100 & 4.75 & 47.87 & 4.92 & 49.53 \\
\hline 12 & 0.79 & 100 & 0.79 & 100 & 0.05 & 6.70 & 0.05 & 5.99 \\
\hline 13 & 0.79 & 100 & 0.79 & 100 & 0.05 & 6.70 & 0.05 & 5.99 \\
\hline
\end{tabular}




\subsection{DER Benefit Analysis with ESS}

According to Section 4.3, the aggregator needs to reduce their generation by the curtailment or shift it by an ESS during the high-NUC period. Thus, an economic benefit analysis was performed for the situation when the ESS is integrated with DERs to shift their generation from high- to low-NUC periods to mitigate congestion issues. The operation of the ESS must be determined without incurring voltage violations and maximizing the profit of the DER simultaneously.

Since the development of the ESS scheduling algorithm is not the main topic here, basic assumptions were made. The charging and discharging efficiencies of the ESS were not considered. The ESS is located at DER 2, which causes a voltage violation and has a greater capacity than DER 1 . The applied ESS capacity is $1 \mathrm{MWh}$ of battery with $0.35 \mathrm{MW}$ of the power conversion systems (PCSs), which is the marginal size to mitigate the voltage violations. Furthermore, the ESS can operate only when the DER output is greater than $10 \%$ of the installed capacity, and the forecasting error of the DER is less than $6 \%$ to fulfill the maximum incentive condition. Based on these assumptions, a genetic algorithm (GA) is adopted to develop the optimal schedule of the ESS. The objective function is defined to maximize the total profit by considering the NUC obtained from the proposed method in Equation (15) with the constraints in Equation (16).

$$
\begin{gathered}
\text { Objective function : } \max (\text { Total Profit })=\max \left(\sum_{t=1}^{24} P_{t} \cdot S M P_{t}-P_{t} \cdot N U C_{t}+I_{t}\right), \\
\text { Constraints }:\left\{\begin{array}{l}
P_{t}=E S S_{\text {output }_{t}}+P V_{\text {output }_{t}} \\
0 \leq S_{t} \leq 100 \% \\
\left|E S S_{\text {output }_{t}}\right| \leq P C S_{\text {size }} \\
P_{t} \leq P_{\text {target }}
\end{array},\right.
\end{gathered}
$$

where $P_{t}$ is the summation of DER and ESS generation. The battery's state of charge at time $t\left(S_{S C}\right)$ cannot exceed the installed capacity of the ESS, and the maximum charging and discharging powers of the ESS $\left(\left|E S S_{\text {output }_{t}}\right|\right)$ cannot exceed the installed capacity of PCSs; the $P_{t}$ needs to be maintained less than $P_{\text {target }}$ which is the minimum generation to cause a voltage violation at the bus.

The ESS operation with DER 2 is shown in Figure 9. The ESS is charged during a high-NUC period from 11 a.m. to 3 p.m. by maintaining $P_{t}$ less than $P_{\text {target }}$ and discharged when the NUC is cheaper from 4 p.m. to 8 p.m. Although it can be discharged at 3 p.m., it begins discharging from 4 p.m. because of the relatively low NUC.

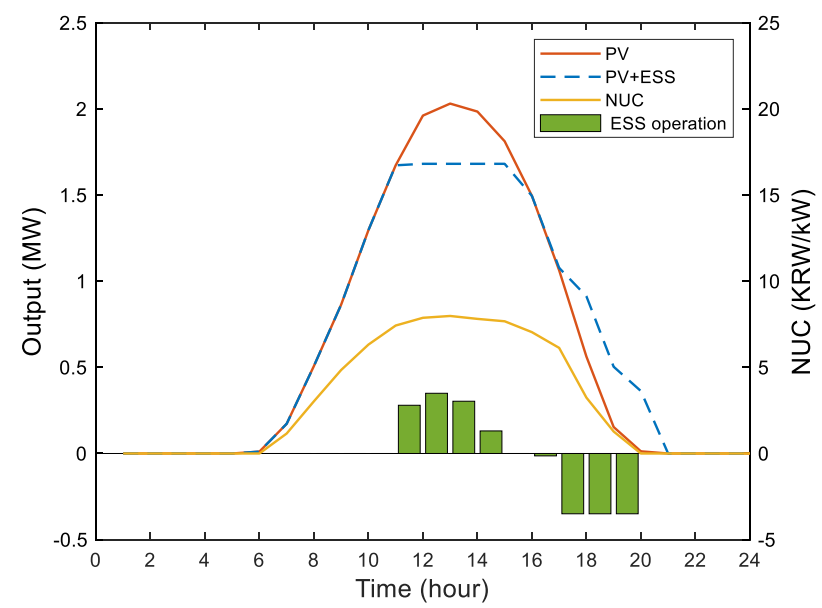

Figure 9. ESS operation with DER 2.

As seen in Table 7, the NUC allocated to DER 2 increases significantly during a high-generation time. However, at other times, when there is no voltage violation, the 
NUC will not vary significantly. Accordingly, the ESS operation was determined. Furthermore, the comparison between the total profit of DER 2 with and without ESS operation shows that the profit varies according to the ESS operation. During the charging time, the profit reduced but increased during the discharging time. However, the total profit in a day increased about KRW 23,185.12 when the DER was operated with the ESS. However, this profit (21.79 KRW/kWh) does not compensate for the wear-out cost of the ESS (500 KRW/kWh) [31].

Table 7. Comparison of total profit of DER 2 with and without ESS operation.

\begin{tabular}{ccccc}
\hline \multirow{2}{*}{ Time } & $\begin{array}{c}\text { NUC } \\
\text { (KRW/kWh) }\end{array}$ & $\begin{array}{c}\text { ESS Operation } \\
\text { (kWh) }\end{array}$ & \multicolumn{2}{c}{ Profit (KRW) } \\
\cline { 4 - 5 } & 0 & 0 & 0 & Without ESS \\
\hline 1 & 0 & 0 & 0 & 0 \\
2 & 0 & 0 & 0 & 0 \\
3 & 0 & 0 & 0 & 0 \\
4 & 0 & 0 & 0 & 0 \\
5 & 0 & 0 & 612.43 & 612.43 \\
6 & 1.17 & 0 & $11,459.75$ & $11,459.75$ \\
7 & 3.08 & 0 & $33,184.73$ & $33,184.73$ \\
8 & 4.94 & 0 & $67,321.18$ & $67,321.18$ \\
9 & 6.45 & 0 & $101,736.49$ & $101,736.49$ \\
10 & 7.55 & 0 & $125,189.76$ & $125,189.76$ \\
11 & 7.95 & 279.96 & $145,586.63$ & $124,808.33$ \\
12 & 8.09 & 349.56 & $109,857.14$ & $97,677.14$ \\
13 & 7.94 & 303.35 & $139,986.79$ & $118,593.08$ \\
14 & 7.79 & 130.98 & $135,876.68$ & $126,058.06$ \\
15 & 7.17 & 0 & $113,808.50$ & $113,808.50$ \\
16 & 6.23 & -13.85 & $82,980.44$ & $85,138.56$ \\
17 & 3.29 & -350 & $46,156.00$ & $74,860.92$ \\
18 & 1.28 & -350 & $12,299.02$ & $40,312.22$ \\
19 & 0 & -350 & 1014.42 & $29,493.92$ \\
20 & 0 & 0 & 0 & 0 \\
21 & 0 & 0 & 0 & 0 \\
22 & 0 & 0 & 0 & 0 \\
23 & 0 & 0 & 0 & 0 \\
24 & & 0 & $1,127,069.96$ & $1,150,255.09$ \\
\hline & & 0 & &
\end{tabular}

To verify the effect of ESS operation owing to the high NUC during the peak time, the voltage profiles at the buses are compared again with and without ESS operation, as shown in Figure 10. In the figure, the ESS operation is able to clear the voltage violations by maintaining the voltage profiles below the limit. It can be concluded that the proposed method is able to allocate the different NUCs at different buses depending on the significance of causing a congestion problem and, thus, encourage the DERs to reduce their generation by charging the ESS to solve the congestion.

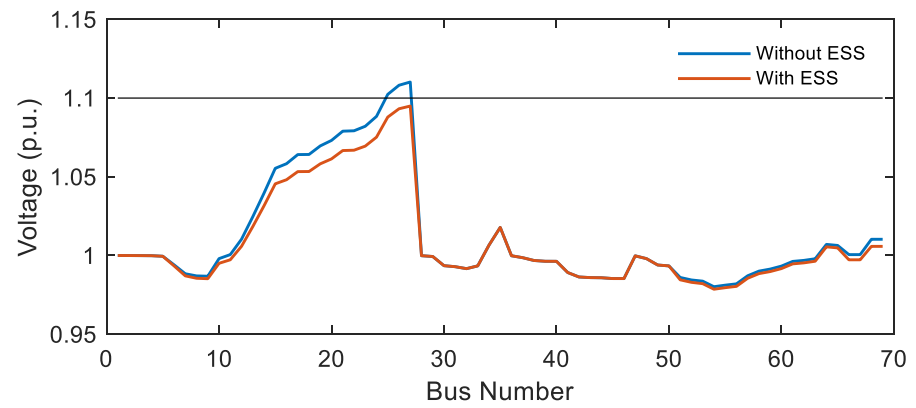

Figure 10. Voltage profile at the buses with and without ESS operation. 


\section{Conclusions}

In this paper, a new approach was proposed for congestion management by allocating different NUCs to DERs who participate in the aggregator market. The sensitivity index was employed to evaluate the congestion risk of DERs in the distribution network. Then, Kirschen's tracing method was modified by using the sensitivity index to distribute the NUC according to the contribution of the DERs to congestion. To verify the effectiveness of the proposed method, the small-scale DER aggregator market in South Korea was simulated using the modified IEEE 69-bus distribution network.

Thus, the proposed method could determine the NUC based on the contribution of each DER to congestion. The DER generation consumed by the local load was charged with a cheaper NUC than the DER generator, which supplied the distant load because it might cause a congestion issue represented by a voltage violation. Furthermore, an economic benefit analysis was performed when the ESS was integrated with DERs to shift their generation from high- to low-NUC periods to mitigate congestion issues. The results showed that ESS utilization could solve congestion problems and reduce the NUC, thus increasing the profits of the aggregator. However, compensation for the ESS operation is not sufficient to overcome the wear-out cost. Hence, additional incentives are required to encourage the installation of ESSs for congestion management in the small-scale DER aggregator market in South Korea.

The findings are expected to provide insight into the congestion management of highpenetration DER distribution systems. Even though the proposed method was evaluated on a small-scale DER aggregator market in South Korea, it takes into account recent research on NCA for various market structures. In future work, economic benefit analysis of the proposed method should be conducted to reflect generalized market regulations.

Author Contributions: Conceptualization, N.N. and J.J.; methodology, N.N., Y.Y. and J.J.; software, N.N. and Y.Y.; validation, Y.Y. and J.J.; formal analysis, N.N., Y.Y. and J.J.; investigation, N.N., Y.Y. and J.J.; resources, J.J. and J.-S.K.; data curation, N.N. and Y.Y.; writing-original draft preparation, N.N., Y.Y. and J.J.; writing-review and editing, N.N., Y.Y. and J.J.; visualization, N.N. and Y.Y.; supervision, J.J. and J.-S.K.; project administration, J.J. and J.-S.K.; funding acquisition, J.J. and J.-S.K. All authors have read and agreed to the published version of the manuscript.

Funding: This work was supported by the Korea Institute of Energy Technology Evaluation and Planning (KETEP) and the Ministry of Trade, Industry \& Energy (MOTIE) of the Republic of Korea (No. 20183010141100). This work was also supported by the "Human Resources Program in Energy Technology" of the Korea Institute of Energy Technology Evaluation and Planning (KETEP) and financially supported by the Ministry of Trade, Industry \& Energy, Republic of Korea (No. 20194030202370).

Conflicts of Interest: The funders had no role in the design of the study; in the collection, analyses, or interpretation of data; in the writing of the manuscript, or in the decision to publish the results.

\section{References}

1. Kirby, B. Congestion Management Requirements, Methods and Performance Indices; Oak Ridge National Laboratory: Oak Ridge, TN, USA, 2002.

2. Pillay, A.S.; Prabhakar, K.; Kothari, D.P. Congestion management in power systems-A review. Int. J. Electr. Power Energy Syst. 2015, 70, 83-90. [CrossRef]

3. Xu, Y.; Sun, H.; Liu, H.; Fu, Q. Distributed solution to DC optimal power flow with congestion management. Int. J. Electr. Power Energy Syst. 2018, 95, 73-82. [CrossRef]

4. Obi, M.; Slay, T.; Bass, R. Distributed energy resource aggregation using customer-owned equipment: A review of literature and standards. Energy Rep. 2020, 6, 2358-2369. [CrossRef]

5. Pantoš, M. Market-based congestion management in electric power systems with exploitation of aggregators. Int. J. Electr. Power Energy Syst. 2020, 121, 106101. [CrossRef]

6. Electric Power Research Institute. Activation of Distributed Energy Resources in the Energy Market; AEMO: Canberra, Australia, 2019.

7. SWECO. Study on the Effective Integration of Distributed Energy Resources for Providing Flexibility to the Electricity System; European Commission: Brussels, Belgium, 2015.

8. Shahidehpour, M.; Yamin, H.; Li, Z. Market Operations in Electric Power Systems: Forecasting, Scheduling, and Risk Management; Institute of Electrical and Electronics Engineers: Piscataway, NJ, USA; Wiley-Interscience: Hoboken, NJ, USA, 2002. 
9. Li, N.; Hakvoort, R.A.; Lukszo, Z. Cost allocation in integrated community energy systems-A review. Renew. Sustain. Energy Rev. 2021, 144, 111001. [CrossRef]

10. Kirschen, D.; Strbac, G. Fundamentals of Power System Economics; Wiley: Hoboken, NJ, USA, 2018.

11. Ahmed, T.; Mekhilef, S.; Shah, R.; Mithulananthan, N.; Seyedmahmoudian, M.; Horan, B. ASEAN power grid: A secure transmission infrastructure for clean and sustainable energy for South-East Asia. Renew. Sustain. Energy Rev. 2017, 67, 1420-1435. [CrossRef]

12. Lima, D.A.; Padilha-Feltrin, A.; Contreras, J. An overview on network cost allocation methods. Electr. Power Syst. Res. 2009, 79, 750-758. [CrossRef]

13. Narimani, M.; Hosseinian, S.H.; Vahidi, B. A modified methodology in electricity tracing problems based on Bialek's method. Int. J. Electr. Power Energy Syst. 2014, 60, 74-81. [CrossRef]

14. Choudhury, N.B.D.; De, M.; Goswami, S.K. Transmission loss allocation in a power market using artificial neural network. Electr. Eng. 2012, 95, 87-98. [CrossRef]

15. Ghadimi, N. Two new methods for power flow tracing using bus power balance equations. J. Cent. South Univ. 2014, 21, 2712-2718. [CrossRef]

16. Strbac, G.; Kirschen, D.; Ahmed, S. Allocating transmission system usage on the basis of traceable contributions of generators and loads to flows. IEEE Trans. Power Syst. 1998, 13, 527-534. [CrossRef]

17. KPX. DER Market and New Business Model; KPX: Jeollanam-do, Korea, 2018.

18. KPX. Energy Market Operating Rules: Amended Version; KPX: Jeollanam-do, Korea, 2020.

19. Jin, H.; Sun, H.; Guo, Q.; Chen, R.; Li, Z. Power system multi-day stochastic scheduling considering the uncertainty of CSP/wind plants. In Proceedings of the 2016 IEEE Power and Energy Society General Meeting (PESGM), Boston, MA, USA, 17-21 July 2016; pp. 1-5. [CrossRef]

20. Kirschen, D.; Allan, R.; Strbac, G. Contributions of individual generators to loads and flows. IEEE Trans. Power Syst. 1997, 12, 52-60. [CrossRef]

21. Malek, J.; Rečka, L.; Janda, K. Impact of German Energiewende on transmission lines in the central European region. Energy Effic. 2017, 11, 683-700. [CrossRef]

22. Eftekharnejad, S.; Vittal, V.; Heydt, G.T.; Keel, B.; Loehr, J. Impact of increased penetration of photovoltaic generation on power systems. IEEE Trans. Power Syst. 2013, 28, 893-901. [CrossRef]

23. Niimura, T.; Niu, Y. Transmission congestion relief by economic load management. In Proceedings of the IEEE Power Engineering Society Summer Meeting, Chicago, IL, USA, 21-25 July 2002; Volume 3, pp. 1645-1649. [CrossRef]

24. Das, D. Optimal placement of capacitors in radial distribution system using a Fuzzy-GA method. Int. J. Electr. Power Energy Syst. 2008, 30, 361-367. [CrossRef]

25. Heo, K.; Kong, J.; Oh, S.; Jung, J. Development of operator-oriented peer-to-peer energy trading model for integration into the existing distribution system. Int. J. Electr. Power Energy Syst. 2021, 125, 106488. [CrossRef]

26. PVWatts Calculator. Available online: https://pvwatts.nrel.gov/pvwatts.php (accessed on 5 April 2021).

27. SMP KPX. Available online: https:/ / www.kpx.or.kr/www/contents.do?key=225 (accessed on 5 April 2021).

28. MATPOWER. Available online: https//matpower.org/ (accessed on 5 March 2021).

29. PG\&E Company. Voltage Tolerance Boundary; PG\&E: San Francisco, CA, USA, 1999.

30. Negash, A.I.; Haring, T.W.; Kirschen, D. Allocating the Cost of Demand Response Compensation in Wholesale Energy Markets. IEEE Trans. Power Syst. 2015, 30, 1528-1535. [CrossRef]

31. Choi, Y.; Kim, H. Optimal scheduling of energy storage system for self-sustainable base station operation considering battery wear-out cost. In Proceedings of the 2016 Eighth International Conference on Ubiquitous and Future Networks (ICUFN), Vienna, Austria, 5-8 July 2016; pp. 170-172. [CrossRef] 